\title{
Sampling strategies for total bacterial count of unpasteurized bulk milk
}

\author{
J. C. F. Pantoja, ${ }^{\star 1}$ G. J. M. Rosa,† D. J. Reinemann, ${ }^{\star}$ and P. L. Ruegg ${ }^{\star}$ \\ ${ }^{*}$ Department of Dairy Science, and \\ †Department of Animal Sciences, University of Wisconsin, Madison 53706
}

\section{ABSTRACT}

The purpose of this study was to assess bulk tank milk sampling strategies for estimating total bacterial count (TBC). Nine large dairies in Wisconsin that produced and shipped at least 1 milk load per day were selected for this study. Total bacteria count was performed for each milk load produced during a 13-mo period. The milk shipment frequency was once $(\mathrm{n}=$ $3)$, twice $(\mathrm{n}=4)$, or 3 times daily ( $\mathrm{n}=2$ farms). A threshold of $8,000 \mathrm{cfu} / \mathrm{mL}$ was used to define increased TBC. The proportion of increased TBC (TBCref) during the study period was defined as the reference probability of an increased TBC for each farm. The number of milk loads that would need to be tested to estimate TBCref precisely (TBCref \pm 0.05 ) in selected time periods (month, quarter, 6 mo, or a year) was calculated assuming independence among TBC measurements. Sampling simulations (systematic or simple random sampling) were used to assess the validity of the independence assumption and compare different sampling schedules (every second, every third, or every seventh milk load) used for estimating TBCref in a 13-mo or 30-d TBC series. The number of milk loads tested to estimate TBCref depended on the time period of interest. For farms with daily milk shipments, at least $94 \%$ of all milk loads produced would need to be tested to estimate TBCref during a $30-\mathrm{d}$ period. In contrast, when the period of interest was a year, reductions of up to $88 \%$ in the number of milk loads tested could be achieved. As the probability of an increased TBC departed from 0.50 toward 1 or 0 , fewer samples were needed to estimate TBCref. A sampling schedule based on TBC performed on every second milk load resulted in $100 \%$ of 5,000 random samples (taken from the 13-mo TBC series) within the range of TBCref \pm 0.05 , indicating that sampling half of the milk loads would precisely estimate TBCref. Results of this study suggest that dairy consultants and processors can adjust the frequency of testing of milk loads depending on the goal of the milk quality monitoring program.

Received October 27, 2011.

Accepted December 29, 2011.

${ }^{1}$ Corresponding author: josecarlospantoja@hotmail.com
Key words: bacterial count, milk quality, sample size, sampling strategy

\section{INTRODUCTION}

A series of indicators have been used to define the quality and safety of unpasteurized bulk tank milk (BTM; Wehr and Frank, 2004). It is desirable that milk is produced with minimal SCC and bacterial counts, no antibiotic or chemical residues, and free of pathogens that can be a threat to human health. Among milk quality indicators, total bacteria count (TBC) has been the reference method adopted by regulatory agencies to ensure the safety and quality of milk (USDA, 2009). When TBC in grade A unpasteurized milk is less than the regulatory limit of $100,000 \mathrm{cfu} / \mathrm{mL}$, it is assumed that all pathogenic and most nonpathogenic bacteria are destroyed by pasteurization.

For regulatory purposes, dairy processors provide state agencies with a randomly selected single monthly TBC result of individual farms. Results of previous studies indicate that the majority of milk loads produced in the United States achieve the regulatory limit for TBC. In 2 studies conducted in New York $(\mathrm{n}=855$ BTM samples and dairies) and Wisconsin ( $\mathrm{n}=7,241$ BTM samples from 16 large dairies), only 5.5 and $2 \%$ of TBC performed were greater than the regulatory limit, respectively (Boor et al., 1998; Pantoja et al., 2009). Nonetheless, milk quality standards adopted by many dairy processors are much more stringent than official regulatory limits because minimizing SCC and TBC dramatically affects the final quality of dairy products (Boor and Murphy, 2002; Barbano et al., 2006).

In Wisconsin, some dairy processors generate large amounts of longitudinal data by performing complete series of bacterial counts for every load of milk produced on the farms. Conversely, some dairy processors perform bacterial counts at longer intervals (e.g., weekly or monthly), or only one or a few counts are performed by independent laboratories as part of milk quality investigations. Regardless of the monitoring schedule, when the purpose of performing bacterial counts is nonregulatory, an outcome of interest is the percent of TBC tested in a sequential series of milk 
loads that are greater than a specific threshold. Such thresholds are arbitrary and commonly defined based on the performance of a certain population of farms. This outcome (percent of increased TBC) has been frequently used by dairy processors and consultants to 1) select farms by assessing bacterial quality of BTM for processing of milk and dairy products, 2) determine milk quality premiums, and 3) diagnose TBC problems (e.g., deviations from normal levels of production).

Based on the cost of acquiring and testing milk samples for intensive monitoring programs, it is relevant to question if fewer samples would generate valid information for the purposes of monitoring and investigating milk quality problems. Conversely, due to the great variation observed for daily bacterial counts (Hayes et al., 2001; Pantoja et al., 2009), the use of one or just a few counts to make inferences about milking hygiene techniques, udder health, and the proper functioning of the milking machine cleaning and sanitation procedures may result in inaccurate conclusions. Determining efficient sampling schedules is, therefore, relevant to reduce costs and yet make valid inferences on the traits being estimated (e.g., proportion of increased TBC in a series of milk loads). The objective of this study was to assess different BTM sampling strategies for estimating TBC.

\section{MATERIALS AND METHODS}

\section{Selection of Farms and Data Collection}

Data from 9 of 16 farms previously enrolled in a separate study were used in this study. Farm selection and data collection for the original study have been described previously (Pantoja et al., 2009). Farms used in the present study were selected based on shipment of at least 1 milk load per day. Milk shipment frequency was once $(\mathrm{n}=3)$, twice $(\mathrm{n}=4)$, or 3 times daily $(\mathrm{n}=$ 2 farms). All enrolled farms shipped milk to a common processor where a series of milk quality tests (TBC, SCC, laboratory pasteurization count, coliform count, and milk temperature) were performed on all milk loads produced from July 2006 to July 2007.

\section{Laboratory Methods}

Microbiological examination of BTM was performed at the dairy processor's laboratory and has been described elsewhere (Pantoja et al., 2009). In brief, TBC was performed using the Petrifilm Aerobic Count (3M Co., St. Paul, MN) method, following standards recommended by the American Public Health Association (Wehr and Frank, 2004).

\section{Statistical Analysis}

The actual number of milk loads shipped per day was determined for each farm. A linear random effects model including the mean $\log _{10}$ TBC $(\mathrm{cfu} / \mathrm{mL})$ as the outcome variable, and day (within week, month, and farm), week (within month and farm), month (within farm), and farm as random terms was constructed to estimate the intraclass correlation (ICC) among observations within the same day, week, month, or farm. The ICC was low $(<0.15)$ for all clusters studied, indicating that the assumption of independence among TBC observations could be assumed as a reasonable approximation for further analyses. Subsequently, a threshold of 8,000 cfu/mL (Pantoja et al., 2009) was used to define increased TBC. The observed proportion of increased TBC (TBCref) during the 13 mo studied was calculated and defined as the reference probability of an increased TBC for each farm. The number of milk loads tested for TBC (sample size) necessary to precisely estimate TBCref for each farm in selected periods of time (month, quarter, 6 mo, or a year) was calculated based on a method for estimating the prevalence of an event in a finite population (Dohoo et al., 2010):

$$
n=\frac{N P(1-P)}{\frac{d^{2}}{Z^{2}(N-1)}+P(1-P)},
$$

where $n$ is the estimated sample size, $N$ is the total number of milk loads shipped and tested for TBC in any given time interval (population size), $P$ is the probability of an increased TBC, $d$ is the confidence limit (defined as \pm 0.05 around the probability of an increased TBC), and $Z$ is the $Z$-score of a standard normal distribution relative to a significance level $(\alpha)$ of 0.05 (2-sided test).

To validate the independence assumption among TBC and assess the accuracy of different sampling schedules in estimating the 13-mo TBCref for each farm, 2 sampling simulation strategies were considered. Systematic sampling was performed using PROC SURVEYSELECT (SAS Institute, 2009) by taking 5,000 samples of the complete TBC series of each farm. Each of the 5,000 samples consisted of equally spaced observations to simulate sampling frequencies that could be adopted in practice when monitoring TBC (e.g., testing of every third milk load, or every other milk load produced). For example, to simulate a weekly BTM sampling schedule for a farm that produced 1 milk load per day, the total number of TBC performed during the 13 mo of study was divided by 7 to obtain the size of the sample. Subsequently, 5,000 samples 
were selected such that the first TBC of the sample was selected at random, and the rest of the sample was completed with equally spaced observations. A histogram was then created using PROC UNIVARIATE (SAS Institute, 2009) to analyze the distribution of TBCref for the 5,000 samples. As a second approach, simple random sampling PROC SURVEYSELECT (SAS Institute, 2009) was performed by randomly selecting each TBC of a given sample from the entire series of data.

When investigating milk quality problems on farms, dairy consultants or processors may be interested in estimating TBCref in shorter time periods to assess deviations from the normal pattern of TBC or to assess the effectiveness of corrective measures. In addition to the sample size calculations performed to estimate TBCref in 30-d periods (Table 1), sampling simulations were repeated using only TBC performed during the first month of the study. In addition to simple random sampling, systematic sampling with sequential observations was also assessed because consultants often have short series of sequential TBC available (e.g., 5 or 7 daily counts in a row). Sequential sampling was performed by selecting the first $\mathrm{TBC}$ at random, and then completing the sample size with sequential observations. For example, if the 10th TBC of the series was selected at random, a sample of size 7 included the 10th to the 16 th TBC.

To validate the independence assumption among TBC by comparing observed (resulting from sampling

Table 1. Milk shipments per day, observed proportion of increased total bacterial count (TBCref), and sample size calculations to estimate the number of milk loads that would need to be tested to detect the observed proportion of increased TBC in loads produced during selected time periods $^{1}$

\begin{tabular}{|c|c|c|c|c|c|c|c|c|}
\hline Farm & $\begin{array}{l}\text { Milk loads } \\
\text { shipped and } \\
\text { tested per day }\end{array}$ & $n^{2}$ & $\begin{array}{l}\text { Number of } \\
\text { increased TBC }\end{array}$ & TBCref & Period & $\begin{array}{l}\text { Number of TBC } \\
\text { performed in } \\
\text { the period }\end{array}$ & $\begin{array}{l}\text { Estimated sample } \\
\text { size to estimate } \\
\text { TBCref } \pm 0.05\end{array}$ & $\begin{array}{c}\text { Reduction }^{3} \\
(\%)\end{array}$ \\
\hline \multirow[t]{3}{*}{ M } & \multirow[t]{3}{*}{0.6} & \multirow[t]{3}{*}{216} & \multirow[t]{3}{*}{66} & \multirow[t]{3}{*}{0.31} & Month & 17 & 16 & 5 \\
\hline & & & & & $6 \mathrm{mo}$ & 100 & 77 & 23 \\
\hline & & & & & Year & 199 & 124 & 38 \\
\hline \multirow[t]{2}{*}{$\mathrm{F}$} & \multirow[t]{2}{*}{0.6} & \multirow[t]{2}{*}{232} & \multirow[t]{2}{*}{46} & \multirow[t]{2}{*}{0.20} & Month & 18 & 17 & 6 \\
\hline & & & & & Year & 214 & 114 & 47 \\
\hline \multirow[t]{4}{*}{ K } & \multirow[t]{4}{*}{0.8} & \multirow[t]{4}{*}{292} & \multirow[t]{4}{*}{104} & \multirow[t]{4}{*}{0.36} & Month & 22 & 21 & 6 \\
\hline & & & & & Quarter & 67 & 57 & 16 \\
\hline & & & & & $6 \mathrm{mo}$ & 135 & 98 & 28 \\
\hline & & & & & Year & 270 & 153 & 43 \\
\hline \multirow[t]{2}{*}{$\mathrm{N}$} & \multirow[t]{2}{*}{1.4} & \multirow[t]{2}{*}{522} & \multirow[t]{2}{*}{201} & \multirow[t]{2}{*}{0.39} & Month & 40 & 36 & 10 \\
\hline & & & & & Quarter & 120 & 91 & 25 \\
\hline $\mathrm{H}$ & 1.4 & 557 & 113 & 0.20 & Year & 514 & 168 & 67 \\
\hline \multirow[t]{4}{*}{$\mathrm{P}$} & 1.7 & 613 & 443 & 0.72 & Month & 47 & 41 & 13 \\
\hline & & & & & Quarter & 141 & 97 & 31 \\
\hline & & & & & $6 \mathrm{mo}$ & 283 & 148 & 48 \\
\hline & & & & & Year & 566 & 200 & 65 \\
\hline $\mathrm{O}$ & 1.7 & 625 & 186 & 0.30 & Month & 48 & 42 & 13 \\
\hline & & & & & Quarter & 144 & 100 & 31 \\
\hline & & & & & $6 \mathrm{mo}$ & 288 & 152 & 47 \\
\hline & & & & & Year & 577 & 207 & 64 \\
\hline I & 2.5 & 909 & 198 & 0.22 & Month & 70 & 55 & 21 \\
\hline & & & & & Quarter & 210 & 117 & 44 \\
\hline & & & & & $6 \mathrm{mo}$ & 420 & 161 & 62 \\
\hline & & & & & Year & 839 & 200 & 76 \\
\hline $\mathrm{E}$ & 2.5 & 957 & 83 & 0.09 & Month & 74 & 46 & 37 \\
\hline
\end{tabular}

${ }^{1} \mathrm{TBC}=$ total bacterial count; increased TBC was defined as counts $\geq 8,000 \mathrm{cfu} / \mathrm{mL}$.

${ }^{2}$ Total number of TBC performed during the 13 mo of the study.

${ }^{3}$ Reduction in the number of milk loads tested in a given period if the calculated sample size was used. 
simulations) and predicted TBCref in both 13- and 1-mo TBC series, the binomial probability mass function (Pagano and Gauvreau, 2000) was used as follows:

$$
P(K=k)=\left(\begin{array}{l}
N \\
k
\end{array}\right) P^{k}(1-P)^{N-k},
$$

where $P(K=k)$ is the probability of observing $k$ increased TBC with probability $P$ (TBCref) in a sample of size $N$. Regardless of the method used for random sampling or sample size calculations, a confidence limit of \pm 0.05 around TBCref was assumed as an acceptable margin of error.

As a final part of the analysis, an adaptive sampling procedure was developed based on principles of process control analysis (Reneau and Lukas, 2006) in which information obtained in the past is used to adapt the procedure. A subset including 2 farms (farms with the greatest and least probability of an increased TBC during the study period) was used to simulate a situation in which a TBC sampling schedule would be initiated on a farm. The procedure consisted of calculating the minimum number of milk loads that would need to be tested to estimate TBCref during an initial 30 -d test period, and then applying the information to future time periods. For a given farm, the number of milk loads tested necessary to estimate TBCref in the first month was calculated using Equation 1 with probability of an increased $\mathrm{TBC}=0.5$ (probability that maximizes the sample size) and assuming that TBC was performed for all milk loads produced on the farm. Subsequently, the probability of an increased TBC observed during the first month, as well as the total number of milk loads tested for TBC were used to calculate the number of milk loads that would need to be tested to estimate TBCref in the following 3-mo period. Likewise, the probability of an increased TBC for the subsequent quarter was derived from the previous quarter so that the sample size would be adjusted using previous information.

\section{RESULTS}

\section{Sample Size}

Different frequencies of TBC testing (equivalent to frequency of milk loads shipped) were observed among farms (Table 1). A total of 4,923 TBC performed on sequential milk loads of 9 farms were included in the analysis. The proportion of increased TBC observed within the entire population of tests was $0.29(\mathrm{n}=$ 1,440). For a hypothetical situation in which TBC was performed on all milk loads, and the farm produced 1 load per day, the number of loads that would need to be tested to precisely estimate the population TBCref (0.29) in time periods of interest was month $=$
$27 ;$ quarter $=70 ; 6 \mathrm{mo}=115$, and year $=170$ milk loads. This represents reductions of $8,22,36$, and $53 \%$, respectively, in the number of loads tested as compared with a schedule in which all loads would be tested.

Sample size calculations indicate that the number of milk loads that would need to be tested to estimate TBCref for each farm varied substantially depending on the period of interest. For farms with approximately daily milk shipments, at least $94 \%$ of all milk loads produced would need to be tested to estimate TBCref within a $30-d$ period (Table 1). When farms shipped milk more frequently (approximately twice or 3 times daily), 63 to $90 \%$ of all milk loads would need to be tested to correctly estimate TBCref within a month. For all farms included in this analysis, the proportion of all milk loads produced that would need to be tested to estimate TBCref decreased when the period of interest increased. When farms were shipping milk 2 to 3 times per day, reductions up to $88 \%$ in the number of loads tested could be achieved if the period of interest was a year (Table 1). Smaller yet substantial reductions (up to $47 \%$ ) were observed for farms where milk loads were shipped once daily.

As expected, the probability of an increased TBC within a farm also influenced the estimated sample size. In general, as the probability of an increased TBC departed from 0.5 toward 0 or 1 , fewer samples were needed to estimate TBCref. For example, the lowest TBCref (0.09) observed among farms would allow the greatest reduction $(88 \%)$ in the number of milk loads tested in a year.

\section{Sampling Simulations}

Results of the sampling simulation (simple random sampling) using the complete 13 -mo TBC series were similar to predictions using the binomial theorem assuming independence among TBC (Table 2) and indicate that, for most farms, reduced testing frequencies would be effective in precisely estimating TBCref (Table 2). Systematic sampling based on TBC performed on alternate milk loads resulted in $100 \%$ of the 5,000 samples falling within the range of TBCref \pm 0.05 , indicating that testing half of the milk loads would precisely estimate TBCref within the 13-mo period. Except for 1 farm, systematic sampling based on TBC performed on every third milk load during the 13-mo period resulted in 66 to $100 \%$ of all 5,000 samples falling within the range of TBCref \pm 0.05 (Table 2). In general, systematic sampling resulted in more precise estimates as compared with simple random sampling. As the size of the samples taken from the TBC series increased, a greater percent of 5,000 samples that resulted in TBCref \pm 0.05 was observed for systematic 
Table 2. Results of different sampling schedules used to estimate the observed proportion of increased total bacterial counts (TBC) within the entire 13-mo series of $\mathrm{TBC}^{1}$

\begin{tabular}{|c|c|c|c|c|c|c|c|c|c|}
\hline \multirow[b]{2}{*}{ Farm } & \multirow[b]{2}{*}{$\mathrm{n}^{2}$} & \multirow[b]{2}{*}{ TBCref $^{3}$} & \multicolumn{2}{|c|}{ Simple random sampling ${ }^{4}$} & \multicolumn{3}{|c|}{ Systematic sampling ${ }^{5}$} & \multicolumn{2}{|c|}{ Binomial prediction ${ }^{6}$} \\
\hline & & & $\begin{array}{c}\text { Sample } \\
\text { size (TBC) }\end{array}$ & $\begin{array}{c}\mathrm{P}(\mathrm{TBCref} \\
\pm 0.05)^{7}\end{array}$ & $\begin{array}{c}\text { Sample } \\
\text { size (TBC) }\end{array}$ & $\begin{array}{l}\text { Sampling } \\
\text { schedule } \\
\text { (milk loads) }\end{array}$ & $\begin{array}{c}\mathrm{P}(\text { TBCref } \\
\pm 0.05)^{7}\end{array}$ & $\begin{array}{c}\text { Sample } \\
\text { size }(\mathrm{TBC})\end{array}$ & $\begin{array}{c}\mathrm{P}(\mathrm{TBCref} \\
\pm 0.05)^{7}\end{array}$ \\
\hline \multirow[t]{3}{*}{ M } & 216 & 0.31 & 31 & 0.43 & 31 & Every 7 th & 0.34 & 31 & 0.56 \\
\hline & & & 72 & 0.73 & 72 & Every 3rd & 0.66 & 72 & 0.63 \\
\hline & & & 108 & 0.87 & 108 & Every other & 1.00 & 108 & 0.75 \\
\hline $\mathrm{F}$ & & & 116 & 0.92 & 116 & Every other & 1.00 & 116 & 0.80 \\
\hline \multirow[t]{3}{*}{$\mathrm{K}$} & 292 & 0.36 & 42 & 0.49 & 42 & Every 7 th & 0.72 & 42 & 0.58 \\
\hline & & & 97 & 0.77 & 97 & Every 3rd & 0.91 & 97 & 0.71 \\
\hline & & & 146 & 0.91 & 146 & Every other & 1.00 & 146 & 0.81 \\
\hline \multirow[t]{3}{*}{$\mathrm{N}$} & 522 & 0.39 & 75 & 0.61 & 75 & Every 7 th & 0.72 & 75 & 0.59 \\
\hline & & & 174 & 0.89 & 174 & Every 3rd & 1.00 & 174 & 0.81 \\
\hline & & & 261 & 0.98 & 261 & Every other & 1.00 & 261 & 0.90 \\
\hline $\mathrm{P}$ & & & 307 & 0.99 & 307 & Every other & 1.00 & 307 & 0.95 \\
\hline \multirow[t]{3}{*}{$\mathrm{O}$} & 625 & 0.30 & 89 & 0.74 & 89 & Every 7 th & 0.61 & 89 & 0.65 \\
\hline & & & 208 & 0.95 & 208 & Every 3rd & 1.00 & 208 & 0.89 \\
\hline & & & 313 & 0.99 & 313 & Every other & 1.00 & 313 & 0.94 \\
\hline \multirow[t]{3}{*}{ I } & 909 & 0.22 & 130 & 0.87 & 130 & Every 7 th & 0.81 & 130 & 0.83 \\
\hline & & & 303 & 0.99 & 303 & Every 3rd & 1.00 & 303 & 0.97 \\
\hline & & & 455 & 1.00 & 455 & Every other & 1.00 & 455 & 0.99 \\
\hline \multirow[t]{3}{*}{$\mathrm{E}$} & 957 & 0.09 & 137 & 0.98 & 137 & Every 7th & 1.00 & 137 & 0.95 \\
\hline & & & 319 & 1.00 & 319 & Every 3rd & 1.00 & 319 & 1.00 \\
\hline & & & 478 & 1.00 & 478 & Every other & 1.00 & 478 & 1.00 \\
\hline
\end{tabular}

${ }^{1}$ Increased TBC were defined as counts $\geq 8,000 \mathrm{cfu} / \mathrm{mL}$.

${ }^{2}$ Total number of TBC performed during the 13 mo of the study.

${ }^{3}$ Proportion of increased TBC observed during the study period.

${ }^{4} 5,000$ samples of size $\mathrm{n}$ taken at random from the entire 13-mo TBC series of each farm.

${ }^{5} 5,000$ samples of size $\mathrm{n}$ taken from the entire 13-mo TBC series. The initial TBC of each sample was selected at random and the remaining observations were equally spaced within the 13-mo period until completion of the sample.

${ }^{6}$ Based on the binomial probability mass function, $\mathrm{P}$ was the probability of a TBC sample of size $\mathrm{n}$ including the proportion of increased TBC observed for each farm during the study period, with a confidence limit of \pm 0.05 .

${ }^{7}$ Proportion of 5,000 samples that fell within the range of TBCref \pm 0.05 .

sampling, as compared with simple random sampling (Figure 1).

In contrast to results of the sampling simulations using the 13-mo TBC series, none of the sampling schedules tested resulted in a precise estimation of TBCref within a 30-d interval (Table 3). Sequential sampling of BTM (especially 3 or 7 TBC performed in a row) resulted in a large proportion of samples that did not include any sample falling within the range of TBCref \pm 0.05 (Table 3 and Figure 2). Except for 1 farm, sampling 15 sequential TBC resulted in a maximum of only $49 \%$ of 5,000 samples falling within TBCref \pm 0.05 .

When sample size calculations were performed according to an adaptive method (Table 4), the number of loads that would need to be tested to estimate TBCref varied substantially among quarters of the year as a response to the probability of an increased TBC observed in the previous period. Farm E was characterized by a much greater TBCref in the first month (0.29), as compared with the subsequent quarters (all $<0.10$; Table 4). The sample size was adjusted as TBCref was updated based on previous periods, which resulted in reductions of 42 to $83 \%$ in the number of milk loads that would need to be tested. As compared with the reference sample size calculations (performed using the actual TBCref observed within a given period), the number of milk loads that would need to be tested per quarter was either under or overestimated depending on the quarter of interest (Table 4).

\section{DISCUSSION}

Intensive monitoring of bacterial counts of BTM is a recent practice that has been increasingly adopted by 

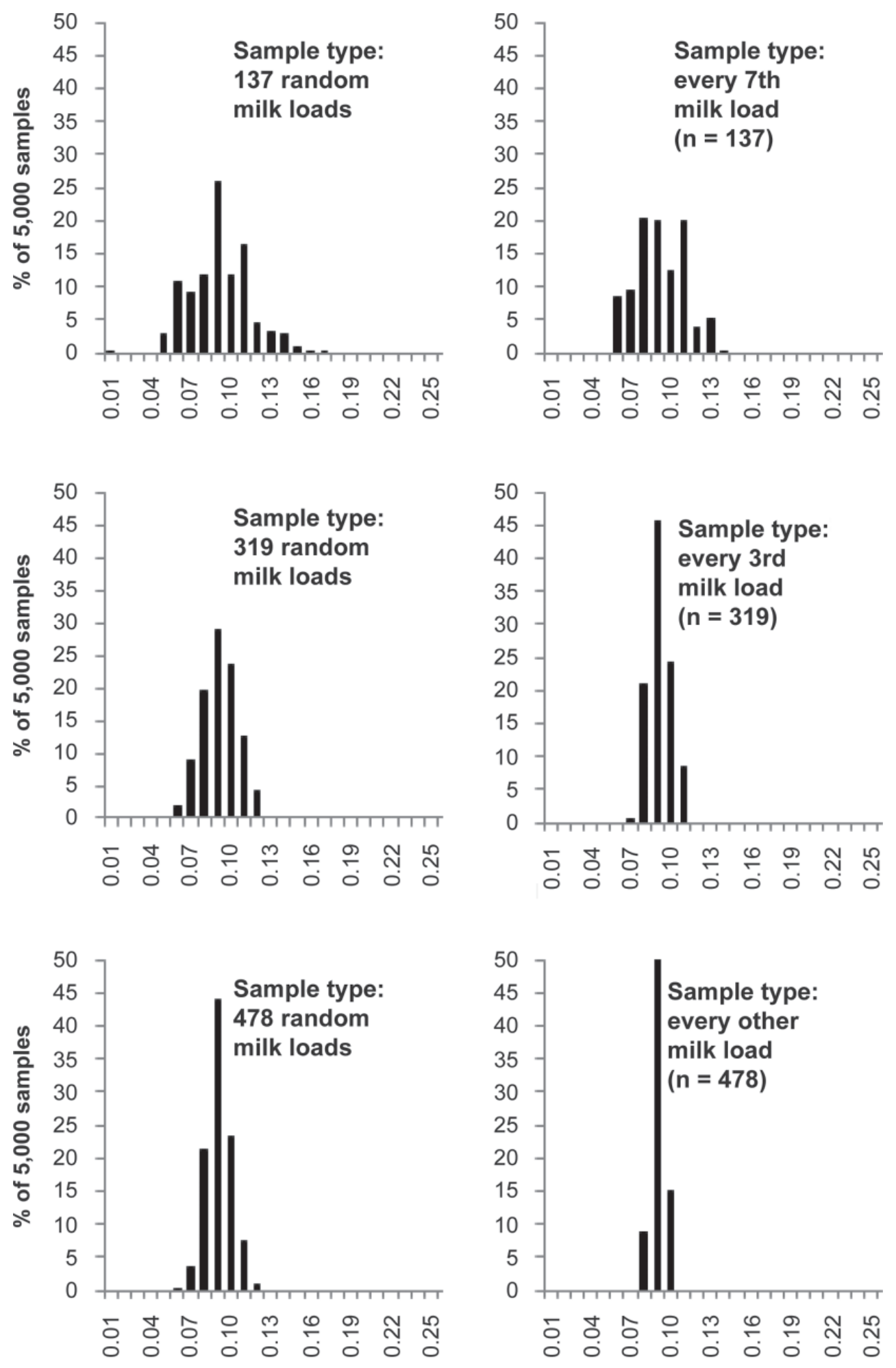

Proportion of increased TBC

per sample

Figure 1. Column on the left: distribution of 5,000 random samples of different sizes taken from the complete annual series of total bacterial count (TBC) for farm E. Column on the right: distribution of 5,000 systematic random samples of different sizes taken from the complete annual series of TBC for the same farm. As the sample size increased, so did the percentage of samples that fell within the observed proportion (0.09) of increased TBC observed for the farm. Increased TBC were defined as counts $\geq 8,000 \mathrm{cfu} / \mathrm{mL}$. 
Table 3. Results of different sampling schedules used to estimate the observed proportion of increased total bacterial counts (TBC) in a 1-mo $\operatorname{period}^{1}$

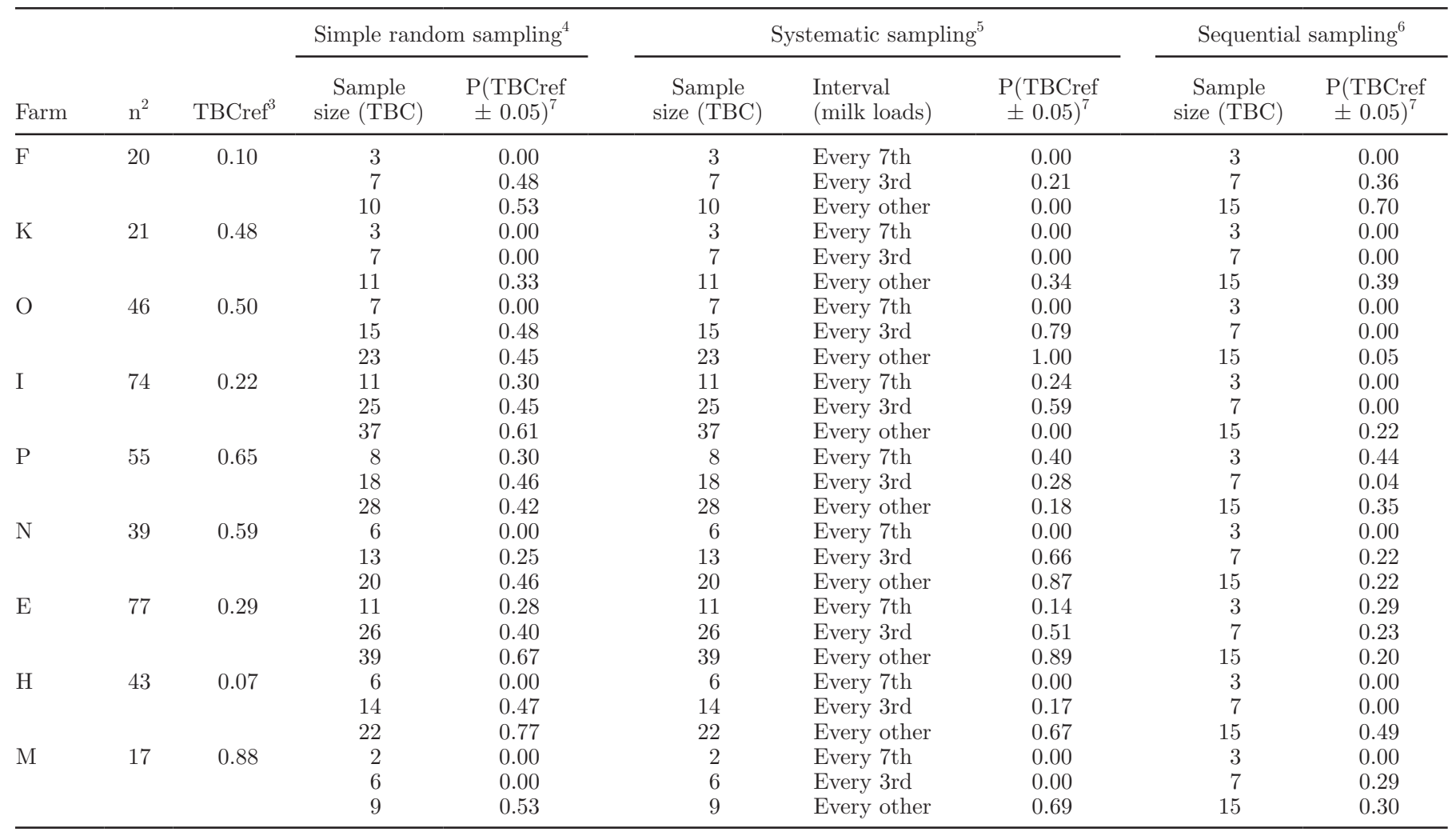

${ }^{1}$ Increased TBC were defined as counts $\geq 8,000 \mathrm{cfu} / \mathrm{mL}$.

${ }^{2}$ Total of TBC performed during the first month of the study.

${ }^{3}$ Proportion of increased TBC observed during the first month of the study.

${ }^{4} 5,000$ samples of size $\mathrm{n}$ taken at random from the first month of the TBC series of each farm.

${ }^{5} 5,000$ samples of size $\mathrm{n}$ taken from the first month of the TBC series of each farm. The initial TBC of each sample was selected at random and the remaining observations were equally spaced within the month until completion of the sample.

${ }_{5}^{6}, 000$ samples of size $\mathrm{n}$ taken from first month of the TBC series of each farm. The initial TBC of each sample was selected at random and sequential observations were selected until completion of the sample.

${ }^{7}$ Proportion of 5,000 samples that fell within the range of TBCref \pm 0.05 .

dairy processors. The understanding and translation of these data into practical actions on the farm depends on the goal of the monitoring program and, in many instances, is still limited due to scarceness of research in this area. By using a longitudinal series of TBC performed on all milk loads produced on the farms, different sampling strategies for TBC could be assessed because a complete series was used as a reference for comparisons.

To interpret results of this study, it is important to emphasize that depending on the objectives of the monitoring program, several outcomes could be used to monitor the TBC of BTM. Examples of such outcomes are the geometric mean TBC within a period of interest, and the use of process control analysis to detect red flags, signaling TBC trends deviating from normal patterns. Although these outcomes may be valuable for specific monitoring purposes, the proportion of increased TBC was chosen as the primary outcome in the present study because it is used by dairy processors to determine milk quality premiums, identify farms based on the bacteriological quality of BTM, and as a practical outcome to diagnose on-farm bacterial count problems. The periods of time (month, quarter, 6 mo, or year) used to calculate the sample size needed to estimate TBCref were chosen because these values could be used by dairy processors to define milk quality premiums or identify farms based on the bacteriological quality of BTM. Likewise, the sampling schedules tested (every seventh, third, or second milk load) are schedules that could be realistic used by processors.

Although simple random sampling was performed to assess the validity of the independence assumption among TBC, dairy processors normally collect BTM 

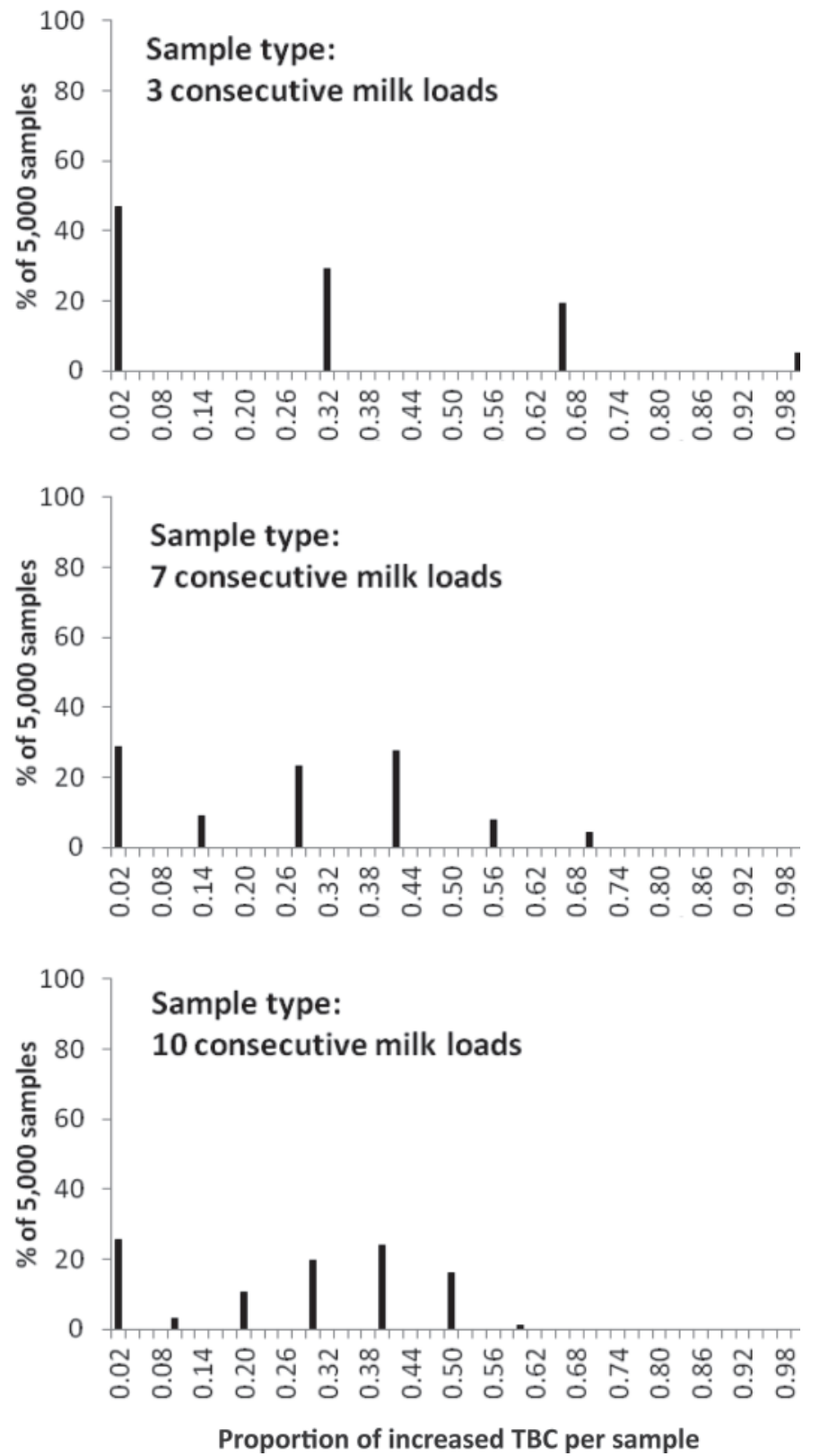

Figure 2. Distribution of 5,000 sequential random samples of different sizes taken from a 30-d (first month of the study) series of total bacterial counts (TBC) performed for Farm E. The observed proportion of increased TBC was 0.29. Increased TBC were defined as counts $\geq 8,000 \mathrm{cfu} / \mathrm{mL}$.

samples in a systematic manner. In fact, systematic sampling appeared to result in greater precision (as compared with simple random sampling) for estimating TBCref within selected periods. This finding could be explained by 2 reasons. By forcing observations to be equally spaced within the entire TBC series, the potential correlation between successive TBC observations within the sample is decreased. In contrast, when TBC were sampled randomly from the same series, samples were more likely to include TBC that were less distant from each other (e.g., 2 consecutive TBC) and, therefore, more correlated to each other. It should be noted that, although weak, some degree of correlation was observed among TBC. Second, the methodology by which the statistical software selects systematic samples from the TBC series (first observation at random and others equally spaced within the series) can result in a great number of identical samples. For example, for a TBC series of size 100, sampling 50 equally spaced TBC (to simulate every other day sampling) would result in only 2 different samples: one consisting of the even numbers (second, fourth, sixth, ... one hundredth), and the other consisting of the odd numbers (third, fifth, seventh, ... ninety-ninth) of the series.

Considering the proportion of increased TBC as the outcome of interest, results suggest that the number of milk loads tested that would be needed to estimate TBCref in a given period of time depends on the goal of the monitoring system. When the goal is to investigate short-term milk quality problems (e.g., increasing bacterial counts due to milking machine cleaning failures), dairy consultants often analyze series of TBC to detect deviations from the normal farm pattern, and assess the outcome of corrective actions. In such situations, results suggest that sampling almost the entire period of interest (e.g., week or month) may be necessary to estimate TBCref within an acceptable range. The use of just a few counts may result in unreliable results (Figure 2). For example, some managers of large dairies monitor TBC performed for multiple milk loads produced on a day to compare effectiveness of premilking preparation performed by different milking technicians. Due to the great variation and multiple risk factors associated with TBC, assessments based on only 1 TBC are likely invalid and should not be encouraged.

In contrast, when the goal is long-term monitoring of bacterial counts (e.g., for determining milk quality premiums), results suggest that performing TBC less often than on every load produced may be sufficient. It was also observed that a greater reduction in the number of milk loads tested for TBC could be achieved for farms that had extreme values of TBCref (approaching 0 or 1 ). This theory was confirmed for both methods using predicted (binomial probability mass function) and observed (sampling simulations) probabilities, and can be important to target farms that could achieve a greater reduction in the rate of TBC testing.

From the standpoint of dairy processors, one of the most important goals for monitoring TBC is to detect milk loads that contain undesirable numbers of bacteria. Milk spoilage caused by bacteria results from different mechanisms, including vegetative growth and presence 
Table 4. Adaptive sampling procedure for total bacterial count (TBC) of bulk tank milk

\begin{tabular}{|c|c|c|c|c|c|c|c|c|c|c|}
\hline Farm & Period & \multicolumn{4}{|c|}{ Adaptive sampling $^{1}$} & \multicolumn{4}{|c|}{ Reference $^{2}$ (actual sampling schedule) } & $\begin{array}{l}\text { Difference in } \\
\text { the number of } \\
\text { samples needed }\end{array}$ \\
\hline \multirow[t]{2}{*}{$\mathrm{P}$} & First month & 55 & 0.50 & 48 & 12 & 55 & 0.65 & 48 & 13 & 0 \\
\hline & 1st quarter & 165 & 0.65 & 112 & 32 & 180 & 0.71 & 115 & 36 & -3 \\
\hline \multirow[t]{5}{*}{$\mathrm{E}$} & First month & 77 & 0.50 & 64 & 17 & 77 & 0.29 & 62 & 19 & 2 \\
\hline & 1st quarter & 231 & 0.29 & 134 & 42 & 228 & 0.08 & 76 & 67 & 58 \\
\hline & 2nd quarter & 228 & 0.08 & 76 & 67 & 214 & 0.03 & 37 & 83 & 39 \\
\hline & $3 \mathrm{rd}$ quarter & 214 & 0.03 & 37 & 83 & 221 & 0.10 & 85 & 61 & -48 \\
\hline & 4th quarter & 217 & 0.10 & 85 & 61 & 217 & 0.06 & 137 & 37 & -52 \\
\hline
\end{tabular}

${ }^{1}$ The sample size needed to estimate the actual proportion of increased $\mathrm{TBC}(\geq 8,000 \mathrm{cfu} / \mathrm{mL})$ in the first month was calculated using Equation 1 , assuming that the probability of an increased TBC was 0.5 (most conservative probability) and that TBC was performed on all milk loads produced on a farm. Subsequently, the actual probability of an increased TBC during the first month as well as the actual number of TBC performed were used to predict these 2 parameters for the first quarter. Likewise, the probability of an increased TBC for the subsequent quarters was estimated based on that of the previous quarter so that the sample size would be adjusted using previous information.

${ }^{2}$ The same procedure described above was repeated using the actual probability of observing an increased TBC (with no time lag) and used as the reference method for comparisons.

${ }^{3}$ Total number of milk loads shipped and tested for TBC.

${ }^{4}$ Probability of an increased TBC observed in the previous period. The initial probability was the most conservative (0.5) and designed to maximize the estimated sample size.

${ }^{5}$ Sample size calculated according to Equation 1 to estimate the actual proportion of increased TBC (with a confidence limit of \pm 0.05 ) in a given period.

${ }^{6}$ Reduction in the number of TBC performed as compared with a schedule in which TBC would be performed for all milk loads produced on the farm.

of spore-forming bacteria that can survive pasteurization (Barbano et al., 2006). Milk components can be degraded by bacteria by means of their metabolism or enzymes (many of which are heat resistant); digestion of proteins and lipids can result in defects in both physical and organoleptic characteristics of milk and dairy products (Barbano et al., 2006). Although thresholds used to define increased TBC are arbitrary and vary among processors, they are useful for purposes of milk quality monitoring and defining milk quality premiums. Thus, processors prefer milk from farms with lower TBCref (such as farm E) so that the frequency of increased $\mathrm{TBC}$ is minimal over time. If decreasing the number of milk loads tested for TBC would precisely estimate TBCref in the long term, the gain resulting from less intensive testing schedules might be considerable.

Payment of milk quality premiums is one strategy that is used to encourage farms to produce milk of high bacterial quality. Although it is common for dairy processors to use TBCref to determine milk quality premiums on a monthly basis (in which case performing TBC on almost all milk loads produced would be necessary), payment systems that precisely estimate TBCref based on less frequent sampling (based on 6-mo or 1-yr period) could be implemented and result in great reductions in the frequency of TBC testing and cost savings for processors. It is important to note that these reductions would apply for processors who aim to monitor industrial bacterial quality of milk. Regulatory TBC testing is normally performed once monthly and because only $2 \%$ of the milk loads produced on the participant farms had TBC greater than $100,000 \mathrm{cfu} / \mathrm{mL}$, sampling schedules for regulatory purposes were not addressed in this analysis for this population of farms.

From the farmers' standpoint, a disadvantage of decreasing the frequency of TBC testing is the longer interval between results. In this case, the system would be less sensitive to detect short-term changes in TBC. This sensitivity of the testing program to detect changes is probably less important for farms that produce milk with outstanding quality because the consistency of milk production practices tends to be in greater control as compared with farms that produce BTM with greater levels of TBC (Reneau and Lukas, 2006). However, for situations in which the time between the test result and the implementation of practical actions needs to be short (e.g., farms that are trying to overcome bacterial count problems or pursuing goals to improve milk quality), a decrease in the rate of TBC sampling could be detrimental. Studies using series of TBC collected dur- 
ing known problematic periods (e.g., diagnosed milking machine wash failures) would be useful for developing alert systems to rapidly detect and respond to deviations from normal patterns of TBC.

Adaptive sampling is a potential monitoring system that would allow a decrease in the long-term TBC sampling frequency, and identify new bacterial counts problems. This method has been applied in different areas such as industrial process control (Reneau and Lukas, 2006) and large clinical trials to adjust the future sample size as participants are enrolled into studies. A simple adaptive sampling schedule such as the one described in this study could be useful not only when TBC monitoring programs are initiated on a farm, but also to adjust the frequency of TBC testing to changes in TBCref. Because dairy processors may have different challenges to sample BTM on a consistent basis, adaptive sampling could be customized to different situations (e.g., the rolling TBCref could be estimated using different periods of time). It should be noted that, for quarters of the year in which the sample size was underestimated (as compared with the reference method), the procedure would lose power to precisely estimate TBCref. As discussed before, this would be detrimental for farms on which monitoring of TBC needs to be done more precisely. Implementation of this method could result in substantial decreases in the cost of sampling and performing TBC, and deserves further investigation.

It is important to emphasize that the analyses performed for this study assumed independence between TBC observations, which, based on available data, was shown to be a good approximation given the low ICC values among observations within various clusters such as days, months, and farms. Nonetheless, sequential bacterial counts produced in populations of farms that are not similar to those used in this study may be characterized by greater correlations among TBC observations. In addition, the threshold used to define an increased TBC was based on the seventy-fifth percentile of the TBC distribution for this population of participant farms. Although this threshold is similar to those used by dairy processors in Wisconsin to determine milk quality premiums, monitoring TBC in different populations may require other meaningful thresholds.

\section{CONCLUSIONS}

Results of this study support that the number of milk loads that would need to be tested to estimate the actual proportion of increased TBC in a given period of time can be adjusted according to the goals of the milk quality monitoring program. For short-term monitoring such as investigation of increased bacterial counts, performing TBC on almost all milk loads produced is important. In contrast, when the goal is to estimate the proportion of increased TBC over long periods of time such as 6 mo or a year, the frequency of TBC testing could be substantially reduced. Adaptive sampling was a useful tool to adjust the number of milk loads tested based on the probability of an increased TBC observed in previous periods.

\section{REFERENCES}

Barbano, D. M., Y. Ma, and M. V. Santos. 2006. Influence of raw milk quality on fluid milk shelf life. J. Dairy Sci. 89(E. Suppl.):E15E19.

Boor, K. J., D. P. Brown, S. C. Murphy, S. M. Kozlowski, and D. K. Bandler. 1998. Microbiological and chemical quality of raw milk in New York State. J. Dairy Sci. 81:1743-1748.

Boor, K. J., and S. C. Murphy. 2002. Microbiology of market milks. Pages 91-122 in Dairy Microbiology Handbook. 3rd ed. R. K. Robinson, ed. John Wiley \& Sons Inc., Hoboken, NJ.

Dohoo, I., W. Martin, and H. Stryhn. 2010. Veterinary Epidemiologic Research. 2nd ed. VER Inc., Charlottetown, Prince Edward Island, Canada.

Hayes, M. C., R. D. Ralyea, S. C. Murphy, N. R. Carey, J. M. Scarlett, and K. J. Boor. 2001. Identification and characterization of elevated microbial counts in bulk tank raw milk. J. Dairy Sci. $84: 292-298$.

Pagano, M., and K. Gauvreau. 2000. Principles of Biostatistics. 2nd ed. Duxbury Press, Belmont, CA.

Pantoja, J. C. F., D. J. Reinemann, and P. L. Ruegg. 2009. Associations among milk quality indicators in raw bulk milk. J. Dairy Sci. 92:4978-4987.

Reneau, J. K., and J. Lukas. 2006. Using statistical process control methods to improve herd performance. Vet. Clin. North Am. Food Anim. Pract. 22:171-193.

SAS Institute. 2009. SAS/STAT 9.2 User's Guide. 2nd ed. SAS Institute Inc., Cary, NC.

USDA (US Food and Drug Administration). 2009. Grade "A" Pasteurized Milk Ordinance, 2009 rev. Accessed Apr. 28, 2010. http:// www.fda.gov/downloads/Food/FoodSafety/Product-SpecificInformation/MilkSafety/NationalConferenceon-InterstateMilkShipmentsNCIMSModelDocuments/UCM209789.pdf.

Wehr, H. M., and J. F. Frank. 2004. Standard Methods for the Examination of Dairy Products. 17th ed. American Public Health Association, Washington, DC. 